\title{
Asthma severity and fertility outcome in women with polycystic ovary syndrome: a registry-based study
}

\author{
Louise Zierau ${ }^{1,2,3}$, Rikke Cortes ${ }^{4}$, Simon Francis Thomsen ${ }^{5,6}$, \\ Espen Jimenez-Solem ${ }^{4,7}$, Svend Lindenberg ${ }^{3}$ and Vibeke Backer ${ }^{1,2}$
}

Affiliations: ${ }^{1}$ Respiratory Research Unit, Bispebjerg Hospital and Frederiksberg University Hospital, Copenhagen, Denmark. ${ }^{2}$ Faculty of Health and Medical Sciences, University of Copenhagen, Copenhagen, Denmark. ${ }^{3}$ Copenhagen Fertility Center, Copenhagen, Denmark. ${ }^{4}$ Dept of Clinical Pharmacology, Bispebjerg Hospital and Frederiksberg University Hospital, Copenhagen, Denmark. ${ }^{5}$ Dept of Dermatology, Bispebjerg Hospital and Frederiksberg University Hospital, Copenhagen, Denmark. ${ }^{6}$ Dept of Biomedical Sciences, Faculty of Health and Medical Sciences, University of Copenhagen, Copenhagen, Denmark. ${ }^{7}$ Dept of Clinical Medicine, Faculty of Health and Medical Sciences, University of Copenhagen, Copenhagen, Denmark.

Correspondence: Louise Zierau, Respiratory Research Unit, Dept of Respiratory Medicine, Bispebjerg University Hospital, Bispebjerg Bakke 23, 2400 Copenhagen NV, Denmark. E-mail: L_Zieraulahotmail.com

ABSTRACT Recent research suggests that women with polycystic ovary syndrome (PCOS) have a higher risk of asthma. However, the severity of asthma, use of antiasthma medication and effect on fertility have yet to be investigated.

In a case-control cross-sectional registry study using the Danish National Patient Register and other Danish registries, asthma prevalence, asthma severity, antiasthma medication use and fertility outcome were investigated among two groups of women with PCOS ( $n=1358$ and $n=17123)$ and a healthy control group $(\mathrm{n}=5340)$.

Both asthma prevalence (OR 1.45, 95\% CI 1.24-1.70) and mean daily inhaled corticosteroid dose were higher among women with PCOS compared with healthy controls, whereas asthma severity was the same in women with and without PCOS. Women with PCOS and asthma had more in vitro fertilisation treatments than women in the control group with asthma, but the numbers of children per woman and spontaneous abortions were the same.

Women with PCOS have a higher prevalence of asthma and a higher use of inhaled corticosteroids, whereas asthma severity is the same in women with and without PCOS. Asthma is associated with more in vitro fertilisation treatments in women with PCOS.

@ERSpublications

Asthma and PCOS are associated; asthma is associated with more in vitro fertilisation treatments in women with PCOS http://ow.ly/BTmh30lTP20

Cite this article as: Zierau L, Cortes R, Thomsen SF, et al. Asthma severity and fertility outcome in women with polycystic ovary syndrome: a registry-based study. ERJ Open Res 2018; 4: 00138-2017 [https://doi.org/10.1183/23120541.00138-2017].

Received: Nov 022017 | Accepted after revision: Sept 112018

Copyright $\odot$ ERS 2018. This article is open access and distributed under the terms of the Creative Commons Attribution Non-Commercial Licence 4.0. 


\section{Introduction}

Polycystic ovary syndrome (PCOS) is the most common endocrine disorder among women of childbearing age, with a prevalence of $9-18 \%$. Additionally, up to $69 \%$ of women with PCOS do not have a formal diagnosis, illustrating the likely existence of a large group of women with undiagnosed PCOS [1]. PCOS is a multifactorial disorder characterised by anovulation, hyperandrogenism, polycystic ovaries, insulin resistance, obesity, infertility, low-grade systemic inflammation and cardiovascular disease [2,3].

Asthma is the most common chronic disease among young adults in the industrialised world, affecting more than 300 million individuals worldwide [4]. Asthma affects 6\% of women of childbearing age and the prevalence increases with obesity $[5,6]$. Asthma is characterised by expiratory airflow limitations with symptoms of wheezing, shortness of breath, chest tightness and cough, with varying symptoms and intensity over time [4].

As with PCOS, asthma is associated with metabolic syndrome [7], especially noneosinophilic asthma in obese women [6], and is also associated with a raised level of systemic inflammation [8]. Moreover, PCOS and asthma are both conditions with a large impact on personal, social and socioeconomic wellbeing, leading to a decreased quality of life $[9,10]$.

The association of PCOS and asthma has been investigated in a few studies [11-13]. Women with PCOS have a significantly higher prevalence of asthma (3\% versus $2 \%$ ), and use significantly more asthma and allergy medicine, than women without PCOS [13]. The higher prevalence of asthma has been found to remain significant even after adjustment for body mass index (BMI) and smoking status [12]. Additionally, women with PCOS and asthma are more often admitted to hospital (10.6\% versus $4.5 \%)$ due to their asthma and experience a higher prevalence (22.8\% versus $14.2 \%)$ of respiratory diseases in general [11]. Impaired fertility is seen in both PCOS and asthma, with a prolonged time to pregnancy [14, 15].

However, little is known with regard to asthma severity among women with PCOS and how asthma affects the fertility of women suffering from PCOS. The aim of the present study was to investigate the prevalence of asthma, use of antiasthma medication and severity of asthma among women with PCOS compared with matched controls, and to explore the association between asthma and fertility among women with PCOS.

\section{Methods}

\section{Study design}

A case-control cross-sectional registry study was performed, consisting of three female cohorts, with data extracted in September 2017 (figure 1).

\section{Data sources}

The data for this study were extracted and merged from the Danish National Patient Register [16], the Danish In Vitro Fertilisation (IVF) Register [17], the Danish National Birth Cohort [18] and the Danish national prescription registries [19]. The unique civil registration number of all the women of the three

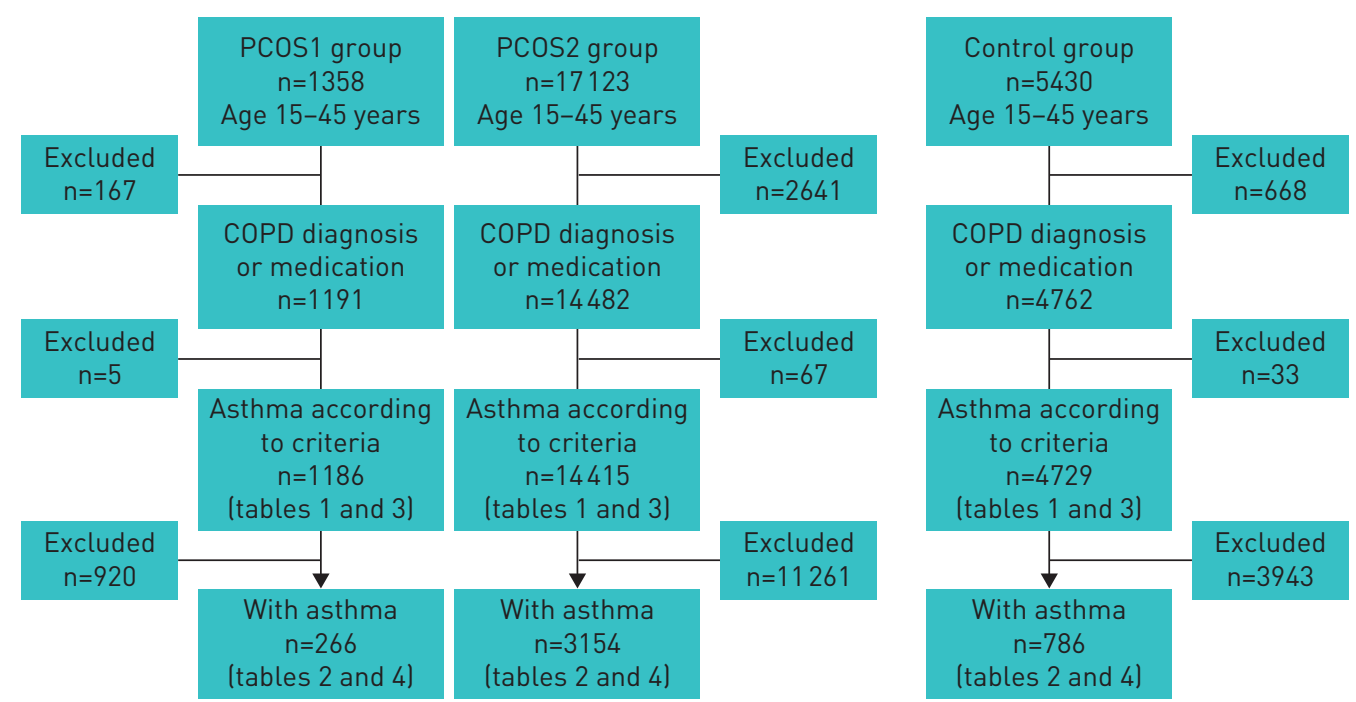

FIGURE 1 Study population. PCOS: polycystic ovary syndrome; COPD: chronic obstructive pulmonary disease. 
groups was used to cross-link data between the different registries. Follow-up on registry data was from 1994 to 2017 (23 years).

\section{Study population}

PCOS1 group

The PCOS1 group consisted of 1358 women diagnosed with PCOS according to the Rotterdam criteria [20]. All the women were diagnosed with PCOS by the same specialist in gynaecology and obstetrics at Copenhagen Fertility Center (Copenhagen, Denmark). A second doctor confirmed the PCOS diagnosis through medical reports and blood samples. All the women in the PCOS1 group were referred with and treated for PCOS in private practice. The youngest was born in 2001 and the oldest was born in 1944.

\section{PCOS2 group}

The PCOS2 group consisted of 17123 women with the International Classification of Diseases (ICD)-10 diagnosis E28.2 (PCOS) from the Danish National Patient Register. None of the patients from the PCOS1 group were included in the PCOS2 group.

\section{Healthy control group}

A total of 5430 non-PCOS women were matched 4:1 with the women of the PCOS1 group on age, sex and geographical region of Denmark in which they lived in at the age of 15 years (index date). These controls did not have any of the following diagnoses under ICD-10: E28.2 (PCOS), E28.2C (polycystic ovaries without anovulation), L68.0 (hirsutism), N91.3 (primary oligomenorrhoea), N91.4 (secondary oligomenorrhoea), N91.5 (oligomenorrhoea unspecified), N91.0 (primary amenorrhoea), N91.1 (secondary amenorrhoea) or N91.2 (amenorrhoea unspecified), or under ICD-8: 626.11 (oligomenorrhoea), 626 (amenorrhoea) or 704 (hirsutism).

Age

Women aged 15-45 years at the time of data extraction were included (figure 1).

\section{Asthma diagnosis}

Subgroups were defined according to the presence of an asthma diagnosis within the two PCOS groups and the control group. An observation period of 365 days was used to map the use of antiasthma medication starting from the date of the first redeemed prescription of medicine from the Anatomical Therapeutic Chemical (ATC) code R03 group (drugs for obstructive airways disease).

The asthma diagnosis was defined as: redeemed two or more prescriptions of drugs with an ATC code from the R03 group during the 365 days of observation [21] and/or at least one of the ICD-10 codes for asthma from the Danish National Patient Register: J45.9 (asthma, unspecified), J45.0 (allergic asthma), J45.1 (nonallergic asthma), J45.8 (mixed asthma) or J46 (acute severe asthma).

Asthma diagnosis was excluded by the presence of one or more of the following: a diagnosis of chronic obstructive pulmonary disease (COPD) (ICD-10: J44), $\geqslant 45$ years of age (to exclude possible noncoded COPD patients) and/or the use of medication specific for COPD (roflumilast).

\section{Classification of asthma severity}

Asthma severity was classified according to the inhaled corticosteroid (ICS) dose of budesonide (or budesonide equivalent) from the Global Initiative for Asthma (GINA) guidelines [4]. Data were collected from the Danish National Patient Register [22]. GINA steps 1-3 (mild to moderate asthma): $\leqslant 400 \mu \mathrm{g} \cdot \mathrm{day}^{-1}$ budesonide (or budesonide equivalent) in combination with a long-acting $\beta_{2}$-agonist (LABA) or short-acting $\beta_{2}$-agonist (SABA), or monotherapy with ICS, or monotherapy with SABA. GINA steps $4-5$ (severe asthma): $>400 \mu \mathrm{g} \cdot \mathrm{day}^{-1}$ budesonide (or budesonide equivalent) and a second controller: LABA, leukotriene antagonist or theophylline and SABA.

\section{Registries}

All Danish citizens have a personal identification number (civil registration number) [23]. This identification number enables data collection and cross-linking of all public registries used herein. The number of women receiving IVF cycles was identified using data from the Danish IVF Register [17]. The numbers of live/still births and singleton/twin births were identified using data from the Danish National Birth Cohort [18]. Additionally, information on BMI and smoking status was only available for the women who had a live or still birth (data collected at the eighth week of pregnancy at the first visit to the general practitioner's office). Women who stopped smoking in the first trimester of pregnancy were considered as smokers [24]. The Danish National Patient Register was used to draw information on all redeemed prescriptions in Danish community pharmacies [19]. The information included the quantity, 
date of redemption, dose and type (ATC code). As all Danish pharmacies are obliged to register all redeemed prescriptions, the Danish National Patient Register includes $97.5 \%$ of all redeemed prescriptions and is therefore assumed to be comprehensive [22].

\section{Ethics}

The Danish Data Protection Agency (2016-41-4561 and 03503 BBH-2015-011) approved the cross-linking of data. In Denmark, ethical approval is not required for retrospective registry-based studies.

\section{Statistics}

The distribution of characteristics was evaluated by mean and standard deviation (range for age) for continuous explanatory variables, and by frequency and within-group percentage for categorical explanatory variables. The descriptive difference between groups was assessed by the p-value of pooled t-tests for continuous variables with equal variance and the Satterthwaite approximation for continuous variables with unequal variance. For categorical variables, a Chi-squared test was used together with Fisher's exact test in the case of antiasthma medication, because of the low numbers involved. Odds ratios were calculated for the association between asthma (yes/no) and PCOS1/PCOS2 and for asthma and PCOS1/controls and presented with 95\% confidence intervals. All p-values were calculated from two-tailed tests $(\mathrm{p} \leqslant 0.05$ statistically significant). All analyses were performed using SAS statistical software version 9.4 (SAS Institute, Cary, NC, USA).

\section{Results}

The study population consisted of 1358 women in the PCOS1 group, 17123 women in the PCOS2 group and 5340 in the control group (figure 1).

\section{Asthma and PCOS}

There were 266 (22.4\%) women with asthma in the PCOS1 group, 3154 (21.9\%) women with asthma in the PCOS2 group and $786(16.6 \%)$ women with asthma in the control group. Among these asthmatic women, those in the PCOS2 group had a higher BMI and more were smokers than among women with asthma in the PCOS1 group. In contrast, the BMI of women with asthma in the PCOS1 group was higher than women with asthma in the control group, which was comprised of more smokers (table 1). SABA antiasthma medication use was the highest among the three groups, followed by the use of ICSs. Overall, the use of antiasthma medication was similar between women with asthma in the three groups, although with a higher use of ICSs in the PCOS1 group compared with the PCOS2 group (table 2).

No difference in the prevalence of asthma was seen between the two PCOS groups. In contrast, a difference in asthma prevalence between the PCOS1 group and the control group was seen (OR 1.45, 95\% CI 1.24-1.70) (table 1).

\section{TABLE 1 Characteristics of women in the study population meeting the inclusion criteria}

\begin{tabular}{|c|c|c|c|c|c|c|c|}
\hline & $\begin{array}{l}\text { PCOS1 } \\
\text { group }\end{array}$ & $\begin{array}{l}\text { PCOS2 } \\
\text { group }\end{array}$ & p-value ${ }^{\#}$ & $\mathrm{OR}^{\#}(95 \% \mathrm{Cl})$ & $\begin{array}{l}\text { Control } \\
\text { group }\end{array}$ & OR ๆ $(95 \% \mathrm{CI})$ & p-value ? \\
\hline Subjects & $1186(5.8)$ & 14415 (70.9) & & & 4729 (23.3) & & \\
\hline BMI available & $694(58.5)$ & $8118(56.3)$ & & & $2685(56.8)$ & & \\
\hline $\mathrm{BMI} \mathrm{kg} \cdot \mathrm{m}^{-2}$ & $25.8 \pm 5.31$ & $26.89 \pm 6.04$ & $<0.001$ & & $24.25 \pm 4.92$ & & $<0.001$ \\
\hline Smoker ${ }^{+}$ & $74(10.4)$ & $1505(17.7)$ & $<0.001$ & & $573(19.6)$ & & $<0.001$ \\
\hline GINA steps $1-3$ ( $\leqslant 400 \mu \mathrm{g} \cdot$ daily $^{-1}$ ICS) & $211(80.5)$ & $2464(79.6)$ & & & 608 (78.8) & & \\
\hline Dose ICS $\mu \mathrm{g} \cdot$ day $^{-1}$ & $148 \pm 107.9$ & $139 \pm 96.0$ & 0.3423 & & $128 \pm 87.9$ & & 0.0527 \\
\hline GINA steps 4-5 (>400 $\mu \mathrm{g} \cdot$ daily $^{-1}$ ICS) & $51(19.5)$ & $617(19.9)$ & & & $158(20.5)$ & & \\
\hline Dose ICS $\mu \mathrm{g} \cdot$ day $^{-1}$ & $625 \pm 482.5$ & $698 \pm 1723.0$ & 0.4527 & & $736 \pm 1029.9$ & & 0.2975 \\
\hline
\end{tabular}

Data are presented as $\mathrm{n}(\%), \mathrm{n}$ (range) or mean \pm SD, unless otherwise stated. PCOS: polycystic ovary syndrome; BMI: body mass index; GINA: Global Initiative for Asthma; ICS: inhaled corticosteroid. BMI and smoking status only available for women who have given birth, data from the Danish National Birth Cohort. ${ }^{\#}$ : difference between PCOS1 and PCOS2; ${ }^{\text {: }}$ : difference between PCOS1 and control; ${ }^{+}$: women with data on smoking status: PCOS1 $n=727$, PCOS2 $n=8336$ and control $n=2943$; ${ }^{\S}$ : test for association between GINA steps and patient group. $p \leqslant 0.05$ statistically significant. 


\begin{tabular}{|c|c|c|c|c|c|}
\hline & PCOS1 group & PCOS2 group & p-value ${ }^{\#}$ & Control group & p-value $e^{\pi}$ \\
\hline Subjects & 266 & 3154 & & 786 & \\
\hline SABA & 231 (88.2) & 2759 (88.9) & 0.7125 & $670(86.56)$ & 0.5048 \\
\hline ICS & $147(56.1)$ & $1468(47.3)$ & 0.0062 & $385(49.7)$ & 0.0748 \\
\hline ICS/LABA & $17(6.5)$ & $282(9.1)$ & 0.1556 & $57(7.4)$ & 0.6342 \\
\hline LAMA/LABA & $0(0)$ & $13(0.4)$ & 0.2938 & $5(0.7)$ & 0.1922 \\
\hline LABA & $12(4.6)$ & $171(5.5)$ & 0.5235 & $54(7.0)$ & 0.1698 \\
\hline LAMA & $0(0)$ & $4(0.1)$ & 0.5609 & $1(0.1)$ & 0.5605 \\
\hline Biological treatment $^{+}$ & $0(0)$ & $0(0)$ & & $0(0)$ & \\
\hline Oral steroid & $6(2.3)$ & 120 (3.9) & 0.1966 & $31(4.0)$ & 0.1960 \\
\hline Local steroid & $1(0.40)$ & $4(0.1)$ & 0.3077 & $0(0)$ & 0.0855 \\
\hline Nasal steroid & $32(12.2)$ & $347(11.2)$ & 0.6122 & $108(14.0)$ & 0.4765 \\
\hline \multicolumn{6}{|c|}{ 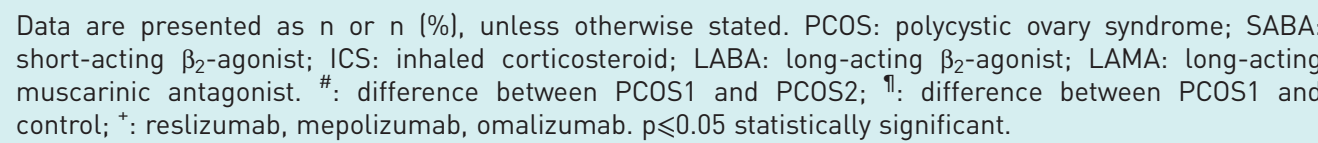 } \\
\hline
\end{tabular}

Among women with asthma, the severity (GINA steps 1-3 or 4-5) of asthma was the same in the two PCOS groups compared with the control group and so was the mean daily ICS dose. The only difference seen between the groups was a higher use of ICSs in the PCOS1 group in GINA steps 1-3 compared with the control group (table 1).

\section{Fertility and PCOS}

The number of women receiving IVF treatment and the number of menstrual cycles before achieving pregnancy within the PCOS1 group were higher compared with the PCOS2 group and the control group. However, more women in the PCOS2 group had a pregnancy and there was a higher number of children per woman than in the PCOS1 group. Among the controls, a higher number of children per woman and more ectopic pregnancies were seen compared with the PCOS1 group; however, women in the PCOS1 group had more twin pregnancies than women in the control group (table 3).

\section{Fertility and PCOS and asthma}

Women with asthma in the PCOS1 group received more IVF treatments than women in the control group and the PCOS2 group. The PCOS1 group also experienced more live births than the PCOS2 group and

\section{TABLE 3 Fertility within the study population meeting the inclusion criteria}

\begin{tabular}{lccccc} 
& $\begin{array}{c}\text { PCOS1 } \\
\text { group }\end{array}$ & $\begin{array}{c}\text { PCOS2 } \\
\text { group }\end{array}$ & p-value & $\begin{array}{c}\text { Control } \\
\text { group }\end{array}$ & p-value \\
\hline $\begin{array}{lcccc}\text { Subjects } \\
\text { Receiving IVF treatment }\end{array}$ & 1186 & 14415 & & 4729 & \\
Menstrual cycles before achieving & $431(36.3)$ & $4256(29.5)$ & $<0.001$ & $379(8.0)$ & $<0.001$ \\
$\quad$ pregnancy & $3.51 \pm 2.8$ & $3.07 \pm 2.4$ & $<0.001$ & $2.8 \pm 2.5$ & $<0.001$ \\
$\begin{array}{l}\text { Pregnant } \\
\text { Live birth }\end{array}$ & $829(69.9)$ & $10462(72.6)$ & 0.0474 & $3350(70.8)$ & 0.5247 \\
Children per woman & $740(62.4)$ & $8605(62.9)$ & 0.7633 & $2973(62.9)$ & 0.0682 \\
Still birth & $1.55 \pm 0.72$ & $1.59 \pm 0.76$ & 0.0361 & $1.63 \pm 0.78$ & $<0.001$ \\
Twins & $6(0.5)$ & $76(0.5)$ & 0.9222 & $16(0.3)$ & 0.3966 \\
Spontaneous abortions & $45(3.8)$ & $437(3.0)$ & 0.1445 & $54(1.1)$ & $<0.001$ \\
Ectopic pregnancies & $73(5.6)$ & $969(6.7)$ & 0.4521 & $270(5.7)$ & 0.5571 \\
& $247(20.8)$ & $3139(21.8)$ & 0.4457 & $1110(23.5)$ & 0.0527
\end{tabular}

Data are presented as $n, n(\%)$ or mean $\pm S D$, unless otherwise stated. PCOS: polycystic ovary syndrome; IVF: in vitro fertilisation. " : difference between PCOS1 and PCOS2; ": difference between PCOS1 and control. $p \leqslant 0.05$ statistically significant. 
had more twins than the control group. No differences were found between the other fertility parameters (table 4).

\section{Discussion}

To the best of our knowledge, this is the first study to investigate the severity of asthma based on the use of antiasthma medication among women with PCOS and the association between asthma and their fertility. Women with PCOS experience more asthma than women without PCOS. Despite the higher prevalence of asthma, severity and antiasthma medication use were the same among women with and without PCOS. In contrast, women with PCOS and asthma received more IVF treatments than women with asthma alone, but the number of children per woman among the three groups was the same. As seen with PCOS and asthma, PCOS alone also affects fertility. Women with PCOS received more IVF treatments and had fewer children per woman than healthy controls. Moreover, women treated for PCOS in private practice (PCOS1) had fewer children per woman than women treated in public hospitals (PCOS2) in spite of more IVF treatments, illustrating a possible difference in the severity of PCOS between these two groups.

\section{Asthma prevalence among women with PCOS}

Our study is in line with previous studies demonstrating a higher prevalence of asthma among women with PCOS. The prevalence in this study of $22 \%$, with asthma defined by the ICD-10 diagnosis or antiasthma medication use, is among the highest described, whereas a previous Danish registry study demonstrated a prevalence of only 3\% when using the ICD-10 codes J44 (COPD) and J45 (asthma) only [13]. Two Australian studies found $10.6 \%$ and $15.2 \%$ asthma prevalence among women with PCOS, which is similar to our findings $[11,12]$.

\section{Asthma severity and antiasthma treatment}

In our study, the prevalence of GINA steps 4-5 asthma, at 20-21\%, is in line with earlier epidemiological studies showing a prevalence of $21-29 \%[25,26]$. This indicates that the severity of asthma among women with PCOS is comparable with the general population. However, use of ICSs between the PCOS1 and PCOS2 groups was different. Furthermore, an earlier Danish registry study of men and women found $45.6 \%$ of asthma patients were receiving ICSs and $68.1 \%$ of asthma patients were receiving SABAs, in contrast to a higher general usage of $56.1 \%$ receiving ICSs and $88.2 \%$ receiving SABAs in our study population [27]. This may indicate a higher use of antiasthma medicine among young women aged 15-45 years than in the general asthma population.

\section{Fertility and PCOS}

In our study, PCOS was associated with impaired fertility, as demonstrated previously [15]. Women with PCOS received more IVF treatments than the controls. Furthermore, PCOS patients treated in private practice (PCOS1 group) had more IVF treatments than those treated at public hospitals (PCOS2 group) (table 3). This may indicate that women treated in private practice have a more severe degree of PCOS

\section{TABLE 4 Fertility in the asthma subpopulation of the study population}

\begin{tabular}{|c|c|c|c|c|c|}
\hline & $\begin{array}{l}\text { PCOS1 } \\
\text { group }\end{array}$ & $\begin{array}{l}\text { PCOS2 } \\
\text { group }\end{array}$ & p-value ${ }^{\#}$ & $\begin{array}{l}\text { Control } \\
\text { group }\end{array}$ & p-value ${ }^{\pi}$ \\
\hline Subjects & 266 & 3154 & & 786 & \\
\hline Receiving IVF treatment & 93 (35.0) & $791(25.1)$ & 0.0004 & 72 (9.2) & $<0.001$ \\
\hline $\begin{array}{l}\text { Menstrual cycles before achieving } \\
\text { pregnancy }\end{array}$ & $3.34 \pm 2.4$ & $3.10 \pm 2.5$ & 0.3674 & $3.04 \pm 2.9$ & 0.489 \\
\hline Pregnant & $192(72.2)$ & $2311(73.3)$ & 0.6956 & $591(75.2)$ & 0.3307 \\
\hline Live birth & $164(61.7)$ & $1703(54.0)$ & 0.016 & $486(61.8)$ & 0.9588 \\
\hline Children per women & $1.61 \pm 0.78$ & $1.58 \pm 0.75$ & 0.4031 & $1.62 \pm 0.80$ & 0.8451 \\
\hline Still birth & $2(0.8)$ & $14(0.4)$ & 0.4801 & $2(0.3)$ & 0.2545 \\
\hline Twins & $12(4.5)$ & $97(3.1)$ & 0.1894 & 12 (1.5) & 0.0048 \\
\hline Spontaneous abortions & $16(6.0)$ & $212(6.7)$ & 0.6554 & $52(6.6)$ & 0.7305 \\
\hline Ectopic pregnancies & $56(21.0)$ & $653(20.7)$ & 0.8968 & 208 (26.5) & 0.0785 \\
\hline
\end{tabular}


with a lower chance of achieving pregnancy naturally. This severity may be illustrated by the higher number of menstrual cycles before achieving pregnancy in this group and the fact that these women have fewer children than women with PCOS treated at public hospitals, in spite of more IVF treatments.

\section{Fertility and asthma and PCOS}

Asthma has been shown to prolong the time to pregnancy among women undergoing IVF treatment [14]. Nevertheless, women with asthma achieve the same numbers of pregnancies and live births as healthy controls [28]. In our study, women with asthma and PCOS received more IVF treatments than women with asthma alone, illustrating how double disease may lead to an even greater impairment of fertility. Yet, with the use of IVF treatment, women with PCOS and asthma have the same number of children per woman as those with asthma alone (table 4). Interestingly, the number of spontaneous abortions was the same among women with asthma with and without PCOS; this may indicate how the impaired fertility may be caused by implantation failure or occur even before implantation.

\section{Strength and limitations}

The strengths of this registry study lie in the quality of the registries used, which have been validated for health and health-related welfare research [29]. In addition, all data in the registries were obtained prospectively and not based on questionnaires or interviews, eliminating the risk of recall bias.

An additional strength of this study is the selection of patients in the PCOS1 group. They were all evaluated by two doctors and diagnosed by the Rotterdam criteria, thereby minimising the risk of misclassification.

The identification of women with asthma according to redemption of antiasthma medication ensures inclusion of all patients treated in private practice and not only those in public hospitals. However, this also represents a limitation of our study, as the Danish National Patient Register does not show the level of adherence. Thus, there is a risk of overestimating the medication used and, therefore, the severity of asthma.

Lastly, we wished to control the possible confounding of obesity (BMI), as obesity is associated with both asthma [6] and PCOS [7]. This was unfortunately not possible due to the low number of women with BMI data.

\section{Conclusions}

A higher prevalence of asthma is seen among women with PCOS. The severity of asthma and antiasthma medication use are the same as for women without PCOS. Women with both PCOS and asthma receive more IVF treatments than women with asthma alone, illustrating a possible additive effect on fertility impairment. However, women with PCOS and asthma end up with the same number of children per woman on average as women with asthma alone.

Acknowledgements: A special thanks to Anna von Bülow (Dept of Respiratory Medicine, Bispebjerg Hospital and Frederiksberg University Hospital, Copenhagen, Denmark) for her great support and help in optimising the method used herein.

Conflict of interest: None declared.

\section{References}

1 March WA, Moore VM, Willson KJ, et al. The prevalence of polycystic ovary syndrome in a community sample assessed under contrasting diagnostic criteria. Hum Reprod 2010; 25: 544-551.

2 Moran L, Teede H. Metabolic features of the reproductive phenotypes of polycystic ovary syndrome. Hum Reprod Update 2009; 15: 477-488.

3 Duleba AJ, Dokras A. Is PCOS an inflammatory process? Fertil Steril 2012; 97: 7-12.

4 Global Initiative for Asthma. Global Strategy for Asthma Management and Prevention. 2017. http://ginasthma.org/ 2017-gina-report-global-strategy-for-asthma-management-and-prevention Date last accessed: August 14, 2017.

5 Kwon HL, Belanger K, Bracken MB. Asthma prevalence among pregnant and childbearing-aged women in the United States: estimates from national health surveys. Ann Epidemiol 2003; 13: 317-324.

6 Haldar P, Pavord ID, Shaw DE, et al. Cluster analysis and clinical asthma phenotypes. Am J Respir Crit Care Med 2008; 178: 218-224.

$7 \quad$ Baffi CW, Wood L, Winnica D, et al. Metabolic syndrome and the lung. Chest 2016; 149: 1525-1534.

8 Holgate ST. Innate and adaptive immune responses in asthma. Nat Med 2012; 18: 673-683.

9 Veltman-Verhulst SM, Boivin J, Eijkemans MJC, et al. Emotional distress is a common risk in women with polycystic ovary syndrome: a systematic review and meta-analysis of 28 studies. Hum Reprod Update 2012; 18: 638-651.

10 Chen H, Gould MK, Blanc PD, et al. Asthma control, severity, and quality of life: quantifying the effect of uncontrolled disease. J Allergy Clin Immunol 2007; 120: 396-402.

11 Hart R, Doherty DA. The potential implications of a PCOS diagnosis on a woman's long-term health using data linkage. J Clin Endocrinol Metab 2015; 100: 911-919. 
12 Htet TD, Teede HJ, de Courten B, et al. Asthma in reproductive-aged women with polycystic ovary syndrome and association with obesity. Eur Respir J 2017; 49: 1601334.

13 Glintborg D, Rubin KH, Nybo M, et al. Morbidity and medicine prescriptions in a nationwide Danish population of patients diagnosed with polycystic ovary syndrome. Eur J Endocrinol 2015; 172: 627-638.

14 Gade EJ, Thomsen SF, Lindenberg S, et al. Fertility outcomes in asthma: a clinical study of 245 women with unexplained infertility. Eur Respir J 2016; 47: 1144-1151.

15 Joham AE, Teede HJ, Ranasinha S, et al. Prevalence of infertility and use of fertility treatment in women with polycystic ovary syndrome: data from a large community-based cohort study. J Women's Health 2015; 24: 299-307.

16 Lynge E, Sandegaard JL, Rebolj M. The Danish National Patient Register. Scand J Public Health 2011; 39: Suppl., $30-33$.

17 Andersen AN, Westergaard HB, Olsen J. The Danish In Vitro Fertilisation (IVF) Register. Dan Med Bull 1999; 46: 357-360.

18 Olsen J. The Danish National Birth Cohort - a data source for studying preterm birth. Acta Obstet Gynecol Scand 2005; 84: 539-540.

19 Gaist D, Sørensen HT, Hallas J. The Danish prescription registries. Dan Med Bull 1997; 44: 445-448.

20 Rotterdam ESHRE/ASRM-Sponsored PCOS Consensus Workshop Group. Revised 2003 consensus on diagnostic criteria and long-term health risks related to polycystic ovary syndrome (PCOS). Hum Reprod 2004; 19: 41-47.

21 Pont LG, van der Werf GT, Denig P, et al. Identifying general practice patients diagnosed with asthma and their exacerbation episodes from prescribing data. Eur J Clin Pharmacol 2002; 57: 819-825.

22 Kildemoes HW, Sørensen HT, Hallas J. The Danish National Prescription Registry. Scand J Public Health 2011; 39: 7 Suppl., 38-41.

23 Pedersen CB, Gøtzsche H, Møller JO, et al. The Danish civil registration system. a cohort of eight million persons. Dan Med Bull 2006; 53: 441-449.

24 Knudsen LB, Olsen J. The Danish Medical Birth Registry. Dan Med Bull 1998; 45: 320-323.

25 Firoozi F, Lemière $\mathrm{C}$, Beauchesne $\mathrm{M}$, et al. Development and validation of database indexes of asthma severity and control. Thorax 2007; 62: 581-587.

26 Nolte H, Nepper-Christensen S, Backer V. Unawareness and undertreatment of asthma and allergic rhinitis in a general population. Respir Med 2006; 100: 354-362.

27 von Bülow A, Kriegbaum M, Backer V, et al. The prevalence of severe asthma and low asthma control among Danish adults. J Allergy Clin Immunol 2014; 2: 759-767.

28 Gade EJ, Thomsen SF, Lindenberg S, et al. Asthma affects time to pregnancy and fertility: a register-based twin study. Eur Respir J 2014; 43: 1077-1085.

29 Thygesen LC, Ersbøll AK. Danish population-based registers for public health and health-related welfare research: introduction to the supplement. Scand J Public Health 2011; 39: 7 Suppl., 8-10. 